OPEN ACCESS

Edited by:

Peng Lei,

West China Hospital, Sichuan

University, China

Reviewed by:

Xize Jia,

Hangzhou Normal University, China

Xiaofen $\mathrm{Ma}$

Guangdong Second Provincial

General Hospital, China

*Correspondence:

Xuejun Liu

bncz@sina.com

Jinping Sun

sunjpdoc1969@sina.com

${ }^{\dagger}$ These authors have contributed equally to this work and share first

authorship

Specialty section:

This article was submitted to

Neurodegeneration,

a section of the journal

Frontiers in Neuroscience

Received: 08 March 2021

Accepted: 13 May 2021

Published: 10 June 2021

Citation:

Wu D, Zhao H, Gu H, Han B,

Wang $Q$, Man X, Zhao R, Liu X and

Sun J (2021) The Effects of rs405509

on $A P O E \& 4$ Non-carriers in

Non-demented Aging.

Front. Neurosci. 15:677823.

doi: 10.3389/fnins.2021.677823

\section{The Effects of rs405509 on APOE 84 Non-carriers in Non-demented Aging}

\author{
Dongpeng $\mathrm{Wu}^{1+}$, Han Zhao't, Huali Gu${ }^{3}$, Bin Han ${ }^{1}$, Qingqing Wang ${ }^{1}, \mathrm{Xu} \mathrm{Man}^{4}$, \\ Renliang Zhao', Xuejun Liu $^{2 *}$ and Jinping Sun ${ }^{1 *}$
}

${ }^{1}$ Department of Neurology, The Affiliated Hospital of Qingdao University, Qingdao, China, ${ }^{2}$ Department of Radiology, The Affiliated Hospital of Qingdao University, Qingdao, China, ${ }^{3}$ Department of Emergency Internal Medicine, The Affiliated Hospital of Qingdao University, Qingdao, China, ${ }^{4}$ Institute of Integrative Medicine, The Affiliated Hospital of Qingdao University, Qingdao, China

Background: There is evidence that the $T$ allele of rs405509 located in the apolipoprotein $\mathrm{E}(A P O E)$ promotor region is a risk factor for Alzheimer's disease (AD). However, the effect of the $T / T$ allele on brain function in non-demented aging is still unclear.

Methods: We analyzed the effects of the rs405509 T/T allele on cognitive performances using multiple neuropsychological tests and local brain function using resting-state functional magnetic resonance imaging (rs-fMRI).

Results: Significant differences were found between $T / T$ carriers and $G$ allele carriers on general cognitive status, memory, and attention $(p<0.05)$. Rs-fMRI analyses demonstrated decreased amplitude of low frequency fluctuation (ALFF) in the right middle frontal gyrus, decreased percent amplitude of fluctuation (PerAF) in the right middle frontal gyrus, increased regional homogeneity $(\mathrm{ReHo})$ in the right cerebellar tonsil and decreased ReHo in the right putamen, and decreased degree centrality (DC) in the left middle frontal gyrus $(p<0.05$, corrected). Furthermore, significant correlations were found between cognitive performance and these neuroimaging changes $(p<0.05)$.

Conclusion: These findings suggest that $\mathrm{T} / \mathrm{T}$ allele may serve as an independent risk factor that can influence brain function in different regions in non-demented aging.

Keywords: $A P O E \varepsilon 4$, Alzheheimer's disease, rs405509, amplitude of low-frequency fluctuations, degree centrality (DC), regional homogeneity (ReHo), fALFF (fractional amplitude of low frequency fluctuations), PerAF

\section{INTRODUCTION}

Alzheimer's disease $(\mathrm{AD})$ is a degenerative disease of the brain characterized by progressive cognitive and behavioral impairment in the elderly and the early stages of old age (Ferri et al., 2005). Apolipoprotein $\mathrm{E}(A P O E)$ is the gene most closely related to the occurrence of $\mathrm{AD}$ (Farrer et al., 1997). Compared with non-carriers, carriers of the $\varepsilon 4$ allele of the APOE gene have been shown to be associated with a higher risk of developing Alzheimer's disease in late-onset families (van der Flier et al., 2011). With the advance of neuroimaging techniques, there is increasing evidence that the $A P O E \varepsilon 4$ allele is related to changes in brain structure and function (Reiman et al., 2004; Heise et al., 2011; Machulda et al., 2011). 
However, other APOE polymorphisms have been proved to affect the occurrence of $\mathrm{AD}$, with the exception of the $\varepsilon 4$ allele. The APOE promotor rs405509, also termed Th1/E47cs or $-219 \mathrm{~T} / \mathrm{G}$, has a substantial impact on the expression of the $A P O E$ gene as well as the development of $\mathrm{AD}$ (Lambert et al., 1998b). The rs405509 According to previous reports, the APOE promotor's T/T allele is a verisimilar risk factor for developing $\mathrm{AD}$ (Beyer et al., 2002). This polymorphism is also associated with myocardial infarction and has become a common risk factor for cardiovascular disease and neurodegenerative disease, particularly in aging (Lambert et al., 2000; Ye et al., 2003).

The rs405509 polymorphism is considered functional. As a regulator of $A P O E$ gene expression, it is likely to regulate specific transcriptional processes (Laws et al., 2003). In different cells, such as neuronal and hepatoma cells, in vitro electrophoretic mobility shift assays, the differential binding activity of two alleles and multiple transcription factors has been evident (Artiga et al., 1998; Lambert et al., 2004; Maloney et al., 2010). After death, biochemical analysis of brain tissue demonstrated its regulatory role in the neural system; T/T genotype was associated with decreased APOE expression (Lambert et al., 1998a, 2005). In a case-control study, compared with the rs $405509 \mathrm{~T} / \mathrm{G}+\mathrm{G} / \mathrm{G}$ group, the rs405509 $\mathrm{T} / \mathrm{T}$ homozygote increased the risk for developing AD (Lambert et al., 2002).

Resting-state functional magnetic resonance imaging (rsfMRI) has become a reliable tool for studying brain function (Biswal et al., 1995; Fox and Raichle, 2007). Zhang et al. (2020) found that $\mathrm{T} / \mathrm{T}$ carriers showed an accelerated agerelated increase in functional activation in the left postcentral gyrus compared with G-allele carriers, which demonstrate that the rs405509 T/T allele of APOE causes an age-related brain functional decline in nondemented elderly people. Ma et al. (2016a) found that the APOE-rs405509 interaction impairs elderly's cognitive performance through brain functional network. Additionally, there are also studies that report rs405509 polymorphism affects brain's structure (Shu et al., 2015; Ma et al., 2016b; Chang et al., 2017). Although most analytical techniques such as graph theory, independent component analysis (ICA), seed-based functional connectivity (FC) have described the function of the brain network, these methods cannot completely address local neural changes. Currently, several approaches have been recommended to describe the local characteristics using rs-fMRI data, that is, regional homogeneity (ReHo; Zang et al., 2004), amplitude of low frequency fluctuation (ALFF; Zang et al., 2007), fractional amplitude of low frequency fluctuation (Zou et al., 2008), percent amplitude of fluctuation (PerAF; Jia et al., 2020) and degree centrality (DC; Buckner et al., 2009).

ReHo is proposed to measure the functional synchronization between a given voxel and its neighbor voxels (Zang et al., 2004). ALFF, defined as the mean amplitude of fluctuations within the range of low frequency, directly characterizes each voxel's spontaneous activity (Zang et al., 2007; Zuo et al., 2010). Fractional amplitude of low frequency fluctuations (fALFF), a ratio of the ALFF within a specific low frequency band to the total BOLD fluctuation amplitude within the full frequency band, can be regarded as a standardized ALFF-like metric at the single voxel level and is theoretically a scale-independent method (Zou et al., 2008). PerAF is the percentage of BOLD fluctuations relative to the mean BOLD signal intensity for each time point and averaging across the whole time series, which has better test-retest reliability, both intra- and interscanners (Zhao et al., 2018; Jia et al., 2020). DC is proposed to evaluate intrinsic functional connectivity across the whole brain to reflect the functional network's architecture in a voxel-wise manner (Buckner et al., 2009). These metrics delineate brain functional properties from distinct perspectives. They have been frequently used to study brain functional abnormalities in various neuropsychiatric disorders (Zang et al., 2007; Wu et al., 2009; Hoptman et al., 2010; Paakki et al., 2010; Liang et al., 2011; Premi et al., 2014; Dai et al., 2015; Ma et al., 2019).

In the present study, we employed rs-fMRI to investigate the rs405509's effects on local brain function in non-demented aging. Considering rs405509 as a promoter that can regulate the expression of APOE, the T to G substitution at rs405509 led to an increase of $169 \%$ in promoter activity. We divided the subjects into two groups by rs405509 genotype (TT vs. GG/GT) for further analyzing the differences in neuroimaging. To better understand the polymorphism's impact on the neural system, it is crucial to enroll non-demented aging individuals, which may help clarify how this polymorphism regulates the risk of developing AD. Specifically, we aimed to determine whether and how rs405509 influences the local function using distinct imaging metrics (ReHo, ALFF, fALFF, PerAF, and DC) and whether these local alterations would be related to the clinical features of the participants. Building on prior literature, we hypothesized that rs405509 $\mathrm{T} / \mathrm{T}$ allele might cause selective degeneration in specific brain regions functionally in the preclinical stage, and such degeneration may lead to changes in the brain's cognitive function.

\section{MATERIALS AND METHODS}

\section{Participants}

A total of 79 non-demented participants were included in the present study. All subjects came from the Beijing Aging Brain Rejuvenation Initiative (BABRI) study, Han Chinese and righthanded. Participants who meet the following criteria were eligible to participate in our research: (1) a score of 24 or higher on the Mini-Mental Status Examination (MMSE); (2) age of 50-80 years; and (3) Clinical Dementia Rating of 0 . The exclusion criteria were as follows: (1) abnormalities in the brain structure (noncerebrovascular injury) revealed by MRI examinations, such as tumors, subdural hematoma, and contusion caused by traumatic brain injury; (2) history of addictions, psychiatric or neurologic disease, or treatments that would affect cognitive function; (3) extensive vessel diseases, such as subcortical or cortical infarcts and watershed infarcts; (4) diseases with white matter lesions, such as multiple sclerosis and normal pressure hydrocephalus; and (5) any contraindications for MRI. The Ethics Committee of The Affiliated Hospital of Qingdao University reviewed and approved the research involving human participants. All subjects gave written informed consent. 


\section{Genotyping}

For each participant, we uniformly genotyped the rs405509 polymorphism using a Custom TaqMan SNP Genotyping Assay (Applied Biosystems, Foster City, CA, United States). Another two SNPs, rs7412 and rs429358, which jointly form the APOE $\varepsilon 2$ (with the rs429358-rs7412 haplotype of T-T), $\varepsilon 3$ (T-C), and $\varepsilon 4$ alleles (C-C), were also genotyped. The sample success rates for all three SNPs were $100 \%$, and the reproducibility of all the genotyping was $100 \%$ according to a duplication analysis of at least $10 \%$ of the genotypes. Based on our sample, the rs405509 polymorphism did not show significant deviations from Hardy-Weinberg equilibrium $(p>0.5)$. We divided all subjects into two groups based on their rs405509 genotype: 44 G-allele (including $20 \mathrm{G} / \mathrm{T}$ and $24 \mathrm{G} / \mathrm{G}$ genotype carriers) and $35 \mathrm{~T} / \mathrm{T}$ carriers.

\section{Neuropsychological Testing}

To assess cognitive functions, all participants received a battery of neuropsychological tests, including Minimum Mental State Examination (MMSE; Folstein et al., 1975), and other representative neuropsychological tests evaluating definite cognitive functions in the study of aging, including: (1) Clock Drawing Test (CDT; Ishiai et al., 1993), (2) Auditory Verbal Learning Test (AVLT) Delayed Recall (Rosenberg et al., 1984), (3) Boston Naming Test (BNT; Knesevich et al., 1986), (4) Trail Making Test A (TMT-A; Gordon, 1972), (5) Trail Making Test B (TMT-B; Gordon, 1972), (6) Symbol Digit Modifying Test (SDMT; Sheridan et al., 2006), and (7) Stroop Color-Word Test (Stroop; Koss et al., 1984).

\section{MRI Data Acquisition}

The resting-state fMRI data were acquired from a GE Signa HDX 3.0 Tesla scanner at the Affiliated Hospital of Qingdao University, China. To reduce head motion and the impact of scanner noise, we used noise-reducing headphones and foam padding. The subjects were instructed to keep awake with their eyes closed and stayed still as much as possible.

The rs-fMRI data were acquired using an echo-planar imaging sequence: 33 axial slices, repetition time $(\mathrm{TR})=2,000 \mathrm{~ms}$, echo time $(\mathrm{TE})=30 \mathrm{~ms}$, slice thickness $=3.5 \mathrm{~mm}$, flip angle $=90^{\circ}$, matrix $=64 \times 64,240$ volumes and field of view $(\mathrm{FOV})=200 \mathrm{~mm} \times 200 \mathrm{~mm}$.

High-resolution T1-weighted images were acquired by using magnetization-prepared rapid gradient-echo (MPRAGE) sequence: 176 sagittal slices, $\mathrm{TR}=1,900 \mathrm{~ms}, \mathrm{TE}=3.44 \mathrm{~ms}$, voxel size: $1 \mathrm{~mm} \times 1 \mathrm{~mm} \times 1 \mathrm{~mm}$, acquisition matrix $=256 \times 256$, slice thickness $=1 \mathrm{~mm}, \mathrm{FOV}=256 \mathrm{~mm} \times 256 \mathrm{~mm}$.

\section{fMRI Data Preprocessing}

Resting-state BOLD fMRI data were preprocessed using Data Processing \& Analysis for Brain Imaging (DPABI_V4.0) (Yan et al., 2016) based on MATLAB R2014a. (1) The first ten time points were removed to allow the subjects to adapt to the scanning environment and the signal to reach equilibrium; (2) the remaining volumes were corrected for the acquisition time delay between distinct slices; (3) realignment was performed to correct the head motion, all participants' translational or rotational motion parameters were less than $2 \mathrm{~mm}$ or $2^{\circ}$ throughout the scanning; frame-wise displacement (FD), which indexes the volume-to-volume changes in head position, is also calculated; (4) in the spatial normalization step, the high-resolution structural images were firstly co-registered to the mean functional images; then we used Diffeomorphic Anatomical Registration Through Exponentiated Lie algebra (DARTEL) algorithm to segment and normalize the co-registered structural images to the Montreal Neurological Institute (MNI) space; finally, the functional images were transformed to the MNI space using the same transformation matrices; (5) smoothing normalized data with a $6 \mathrm{~mm}$ full-width at half maximum(FWHM) Gaussian kernel; (6) linear detrending; (7) several nuisance covariates (head motion effect based on the Friston-24 model, the spike volumes with $\mathrm{FD}>0.5$, the white matter signal, and the cerebrospinal fluid signal) were regressed out from the data (Friston et al., 1996; Power et al., 2012); (8) the data were then band-pass filtered $(0.01-0.08 \mathrm{~Hz})$. No preprocessing filtering used for ALFF and fALFF. The fMRI data for ReHo and DC calculation were not smoothed during the pre-processing procedure.

\section{ALFF Analysis}

After preprocessing, the time courses were converted to the frequency domain using a fast Fourier transform, and the power spectrum was obtained by square-rooted fast Fourier transform and averaged across $0.01-0.08 \mathrm{~Hz}$ at each voxel. The averaged square root was considered as the ALFF. To decrease global effects of variability across subjects, the ALFF of each voxel was further divided by the global mean ALFF.

\section{fALFF Analysis}

After obtaining the power spectrum, the square root was calculated at each frequency of the power spectrum and the mean square root was acquired across $0.01-0.08 \mathrm{~Hz}$ band for each voxel. At last, fALFF in each voxel was divided by mean fALFF of the global brain within a full brain mask to standardize for voxels in the whole brain.

\section{PerAF Analysis}

The PerAF of each voxel was calculated as follows,

$$
\begin{gathered}
\operatorname{PerAF}=\frac{1}{n} \sum_{i=1}^{n}\left|\frac{X_{i}-\mu}{\mu}\right| \times 100 \% \\
\mu=\frac{1}{n} \sum_{i=1}^{n} X_{i}
\end{gathered}
$$

where $X_{i}$ is the signal intensity of the $i$ th time point, $n$ is the total number of time points of the time series, and $\mu$ is the mean value of the time series.

\section{ReHo Analysis}

The functional data without spatial smoothing were used to calculate ReHo. Kendall's coefficient of concordance (KCC) was 
used to calculate the synchronization of the time series between a given voxel and its nearest neighbors.

$$
W=\frac{\sum\left(R_{i}\right)^{2}-n(\bar{R})^{2}}{\frac{1}{12} K^{2}\left(n^{3}-n\right)}
$$

where $W$ is the KCC among given voxels, which ranges from 0 to $1 ; R_{i}$ is the sum rank of the time point; $\bar{R}$ is the average value of $R_{i} ; n$ represents the length of time series, and $K$ is the size of the current cluster $(K=27)$. To achieve standardization, the ReHo value of each voxel was then divided by the global mean ReHo value. Ultimately, the ReHo maps spatially smoothed with a $6 \mathrm{~mm}$ FWHM Gaussian kernel to reduce noise.

\section{Analysis}

Degree centrality computation procedure was conducted using fMRI data without smoothing. Pearson's correlation coefficients were calculated between each voxel and each other voxel in the entire brain, and a gray matter functional connectivity matrix for each subject was acquired (Zuo et al., 2012). For a given voxel, weighted DC was calculated as the sum of FC over a threshold of 0.6 between that voxel and all other voxels within the entire gray matter. Next, the weighted DC of each voxel was divided by the global mean weighted DC of each subject to achieve standardization. Finally, we spatially smoothed the weighted DC maps with a $6 \mathrm{~mm}$ FWHM Gaussian kernel. We also analyzed weighted DC differences between $\mathrm{T} / \mathrm{T}$ carriers and $\mathrm{G}$ allele carriers across different r-value thresholds. The specific details are in the Supplementary Materials.

\section{Statistical Analysis}

The differences between T/T carriers and G-allele carriers in age and education were tested with two-sample $t$-tests. The gender difference was examined with the Pearson Chi-Square test.

Two sample $t$-tests were applied to compare the ALFF, fALFF, PerAF, ReHo, and DC maps between T/T carriers and G-allele carriers. To reduce potential effects on the results, we included individual sex, age, education and mean FD as nuisance covariates. Multiple comparisons were corrected using a Gaussian Random Field (GRF) correction with a clusterdefining threshold of $p<0.001$ and a corrected significance of $p<0.05$ in the cluster level. The above-described statistical analyses were done using DPABI (Yan et al., 2016). If any measure exhibited a between-group difference in a cluster (defined as a region of interest [ROI]), a Pearson correlation analysis was performed in all subjects to evaluate its associations with the neuropsychological test scores in an ROI-wise manner. At the threshold of $p<0.05$, the correlation was considered significant.

\section{RESULTS}

\section{Cognitive Characteristic}

The demographic and neuropsychological data are presented in Table 1. There were no significant differences in age, gender, or educational years between rs405509 T/T genotype and G-allele carriers groups.

Compared with the G-allele carriers, the T/T allele carriers showed decreased MMSE $(p<0.001)$, AVLTDelayed Recall $(p=0.014)$, AVLT-T $(p=0.032)$, and SDMT $(p=0.025)$, indicating a decline in the general cognitive status, memory, and attention.

TABLE 1 | Demographic and neuropsychological data of the sample.

\begin{tabular}{lcccc}
\hline Characteristics & T/T carrier & G-allele carrier & Statistics & $\boldsymbol{p}$ Value \\
\hline Number of subjects & 35 & 44 & & \\
Age(years) & $65.31 \pm 2.39$ & $64.80 \pm 2.38$ & $\mathrm{t}=0.962$ & $0.339^{a}$ \\
Education(years) & $8.83 \pm 2.69$ & $9.48 \pm 1.53$ & $\mathrm{t}=-1.270$ & $0.210^{a}$ \\
Gender(M/F) & $15 / 20$ & $19 / 25$ & $\mathrm{x}^{2}=0.001$ & $0.977^{b}$ \\
General cognition & & & & \\
MMSE & $25.20 \pm 1.02$ & $28.57 \pm 1.11$ & $\mathrm{t}=13.879$ & $<0.001^{a}$ \\
Memory & & & & \\
AVLT-Delayed Recall & $3.74 \pm 1.07$ & $4.34 \pm 1.03$ & $\mathrm{t}=-2.520$ & $0.014^{a}$ \\
AVLT-T & $22.00 \pm 2.98$ & $23.75 \pm 4.13$ & $\mathrm{t}=-2.184$ & $0.032^{a}$ \\
ROCF-delay recall & $13.74 \pm 3.45$ & $14.41 \pm 2.96$ & $\mathrm{t}=0.923$ & $0.359^{a}$ \\
Attention & & & & \\
SDMT & $28.20 \pm 8.87$ & $33.59 \pm 11.47$ & $\mathrm{t}=-2.288$ & $0.025^{a}$ \\
TMT-A time(s) & $76.43 \pm 29.56$ & $67.75 \pm 14.87$ & $\mathrm{t}=1.584$ & $0.120^{a}$ \\
Executive Function & & & & \\
SCWT-C time & $78.94 \pm 27.68$ & $74.84 \pm 20.13$ & $\mathrm{t}=0.736$ & $0.465^{a}$ \\
SCWT-C right & $43.89 \pm 5.11$ & $45.02 \pm 4.15$ & $\mathrm{t}=-1.066$ & $0.290^{a}$ \\
TMT-B time(s) & $203.20 \pm 72.60$ & $186.41 \pm 57.19$ & $\mathrm{t}=1.150$ & $0.254^{a}$ \\
Language & & & & \\
BNT & $21.66 \pm 2.66$ & $22.18 \pm 2.71$ & $\mathrm{t}=-0.863$ & $0.391^{a}$ \\
\hline
\end{tabular}

The data are shown as the mean \pm standard deviation. $M$, male; F, female; MMSE, Mini-Mental State Examination; AVLT, Auditory Verbal Learning Test; AVLT-T, AVLT-total; ROCF, Rey-Osterrieth Complex Figure; SDMT, Symbol Digit Modalities Test; TMT, Trail Making Test; SCWT, Stroop Color and Word Test; BNT, Boston Naming Test.

${ }^{a}$ The $p$ values were obtained by two-sample t-tests.

${ }^{b}$ The $p$ value was obtained by chi-square test.

TABLE 2 | Brain regions showing ALFF, PerAF, ReHo, and DC differences between the two groups.

\begin{tabular}{lcccccc}
\hline & & & \multicolumn{3}{c}{ MNI coordinate (mm) } \\
\cline { 5 - 6 } Regions & $\begin{array}{c}\text { Cluster size } \\
\text { (voxels) }\end{array}$ & $\begin{array}{c}\text { Peak T } \\
\text { value }\end{array}$ & $\mathbf{X}$ & $\mathbf{Y}$ & $\mathbf{Z}$ \\
\hline ALFF & 114 & -5.5769 & 30 & 36 & 30 \\
$\begin{array}{l}\text { Middle Frontal Gyrus (R) } \\
\begin{array}{l}\text { PerAF } \\
\text { Middle Frontal Gyrus (R) }\end{array}\end{array}$ & 58 & -4.7067 & 30 & 36 & 30 \\
$\begin{array}{l}\text { ReHo } \\
\text { Cerebellar Tonsil (R) }\end{array}$ & 79 & 5.4451 & 12 & -48 & -45 \\
$\begin{array}{l}\text { Putamen (R) } \\
\text { DC }\end{array}$ & 69 & -4.8474 & 30 & -3 & -3 \\
Middle Frontal Gyrus (L) & 83 & -4.9383 & -36 & 33 & 30
\end{tabular}

ReHo, regional homogeneity; ALFF, amplitude of low frequency fluctuation; PerAF, percent amplitude of fluctuation; $D C$, degree centrality; $L$, left; $R$, right. 
TABLE 3 | Relationship between local neuroimaging metrics and cognitive performance in a ROI-based correlation analysis.

\begin{tabular}{lcccc}
\hline \multicolumn{1}{c}{ ALFF } & \multicolumn{2}{c}{ ReHo } & DC \\
\cline { 3 - 4 } $\begin{array}{l}\text { Cognitive } \\
\text { tests }\end{array}$ & $\begin{array}{c}\text { Right Middle } \\
\text { Frontal Gyrus }\end{array}$ & $\begin{array}{c}\text { Right } \\
\text { Cerebellar } \\
\text { Tonsil }\end{array}$ & $\begin{array}{c}\text { Right } \\
\text { Putamen }\end{array}$ & $\begin{array}{c}\text { Left Middle } \\
\text { Frontal Gyrus }\end{array}$ \\
\hline MMSE & $r=0.448$ & $\begin{array}{c}r=-0.417 \\
p<0.001^{*}\end{array}$ & $\begin{array}{c}r=0.491 \\
p<0.001^{*}\end{array}$ & $\begin{array}{c}r=0.404 \\
p<0.001^{*}\end{array}$ \\
\hline
\end{tabular}

ReHo, regional homogeneity; ALFF, amplitude of low frequency fluctuation; DC, degree centrality; ROI, region of interest; MMSE, Mini-Mental State Examination. ${ }^{*} p<0.05$.

\section{Intergroup Differences in ALFF, fALFF, PerAF, ReHo, and DC}

Compared with G-allele carriers, T/T carriers exhibited decreased ALFF in the right middle frontal gyrus (Table 2 and Figure 1), decreased PerAF in the right middle frontal gyrus (Table 2 and Figure 2), increased $\mathrm{ReHo}$ in the right cerebellar tonsil and decreased ReHo in the right putamen (Table 2 and Figure 3), and decreased DC in the left middle frontal gyrus (Table 2 and Figure 4) $(p<0.05$, GRF corrected). For fALFF, no cluster survived after multiple comparisons ( $p>0.05$, GRF corrected).

\section{Correlations Between Neuroimaging Parameters and Cognitive Scores}

We conducted correlation analyses between ROI-based mean imaging values and cognitive scores. Correlations were found between MMSE and imaging parameters in significant ROIs of ALFF, ReHo, and DC (Table 3 and Figure 5). No significant correlation was found for other cognitive measures and other local metrics.

\section{DISCUSSION}

The ALFF, fALFF, PerAF ReHo, and DC represent the local brain function from distinct aspects. These local metrics can identify regional brain abnormalities with great sensitivity. In the current study, we used these local metrics to find local brain functional differences between the $\mathrm{T} / \mathrm{T}$ carriers and $\mathrm{G}$ allele carriers and further analyzed the relationship of these functional alterations with neuropsychological scores. The results showed that compared with the $G$ allele carriers, the $\mathrm{T} / \mathrm{T}$ carriers manifested decreased ALFF in the right middle frontal gyrus, decreased PerAF in the right middle frontal gyrus, increased ReHo in the right cerebellum posterior lobe, decreased ReHo in the right putamen, and decreased DC in the left middle frontal gyrus. These findings may improve our understanding of the influence of rs405509 on the functional changes of $A P O E \& 4$ non-carriers.

Amplitude of low frequency fluctuation reflects the degree of spontaneous neural activity (Zang et al., 2007). In the current study, the right middle frontal gyrus of $\mathrm{T} / \mathrm{T}$ carriers showed decreased ALFF, which represented reduced spontaneous neural activity within this area. The middle frontal gyrus is thought to be involved in episodic memory retrieval (Buckner et al.,

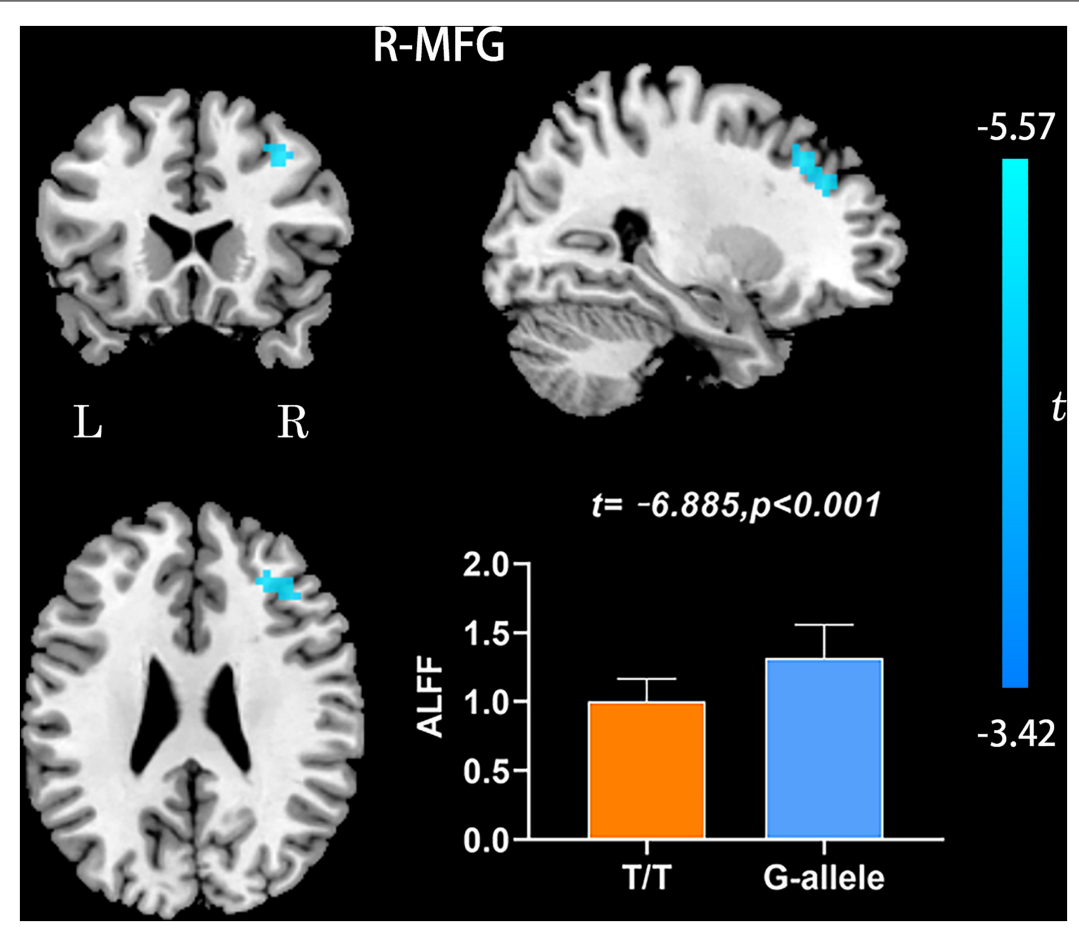

FIGURE 1 | ALFF differences between T/T carriers and G-allele carriers. Error bar represents the standard deviation. ALFF, amplitude of low frequency fluctuation; L, left; $R$, right. 


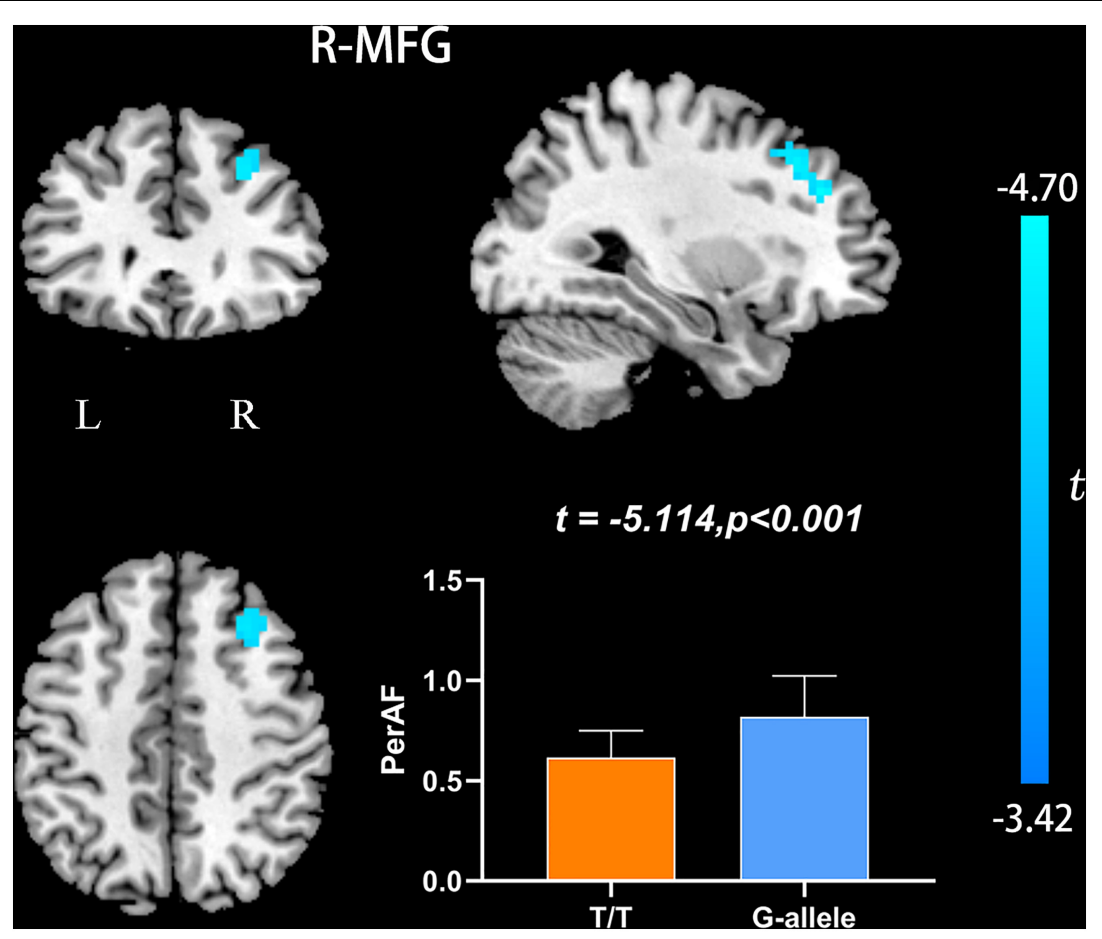

FIGURE 2 | PerAF differences between T/T carriers and G-allele carriers. Error bar represents the standard deviation. PerAF, percent amplitude of fluctuation; L, left; $R$, right.

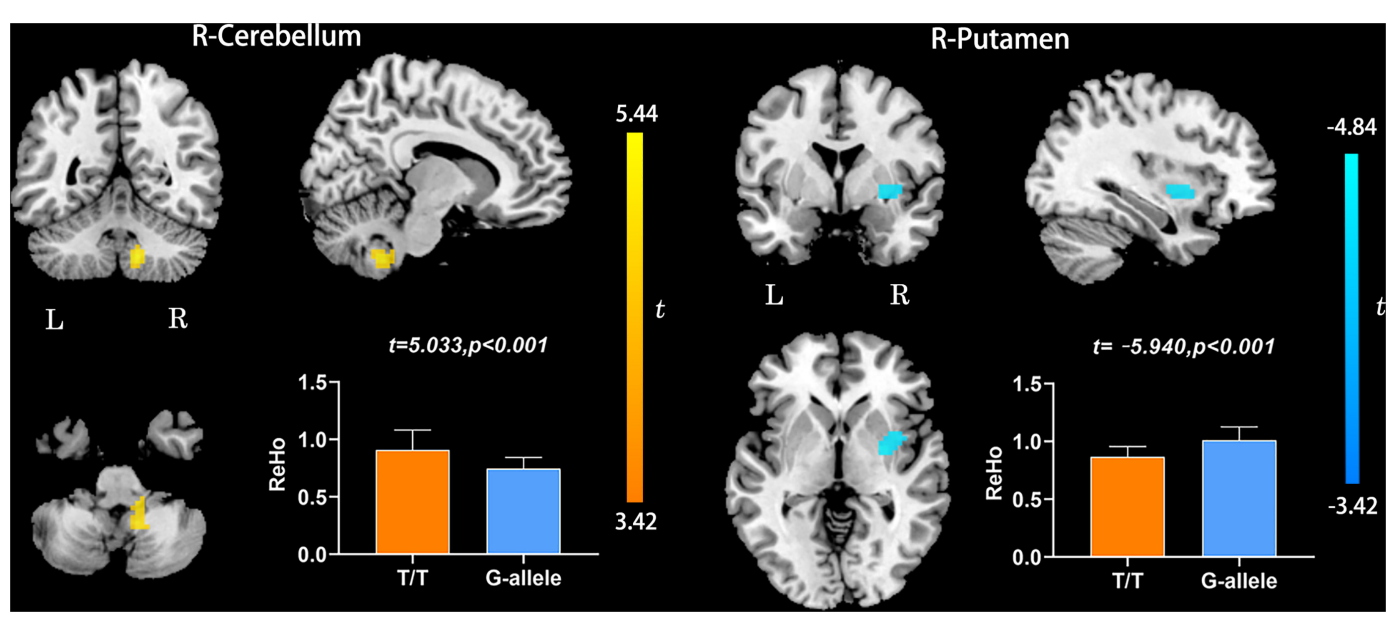

FIGURE 3 | ReHo differences between T/T carriers and G-allele carriers. Error bar indicates the standard deviation. Abbreviations: ReHo, regional homogeneity; L, left; R, right.

2000; West, 2000; Corbetta et al., 2008), and the performance of neuropsychological tests in $\mathrm{T} / \mathrm{T}$ carriers also confirmed the fMRI results. Meanwhile, the right middle frontal gyrus may act as a critical node of the ventral and dorsal networks (Fox et al., 2006). Therefore, we speculate that the reduced local neural activity in the right middle frontal gyrus could account for the decrease of memory and attention. In the current study, we also found decreased PerAF in the right middle frontal gyrus, consistent with the ALFF result. PerAF has better test-retest reliability than conventional ALFF and much better than fALFF, both intra- and inter-scanners (Zhao et al., 2018; Jia et al., 2020). However, for the fALFF analysis, no cluster survived after multiple comparisons $(p>0.05$, GRF corrected). Compared with ALFF, previous researches have shown that fALFF had lower test-retest reliability in gray matter voxels (Zuo et al., 2010; Küblböck et al., 2014). Consequently, fALFF may not be a suitable standardized metric for frequency-specific studies. For a single voxel, PerAF is a 


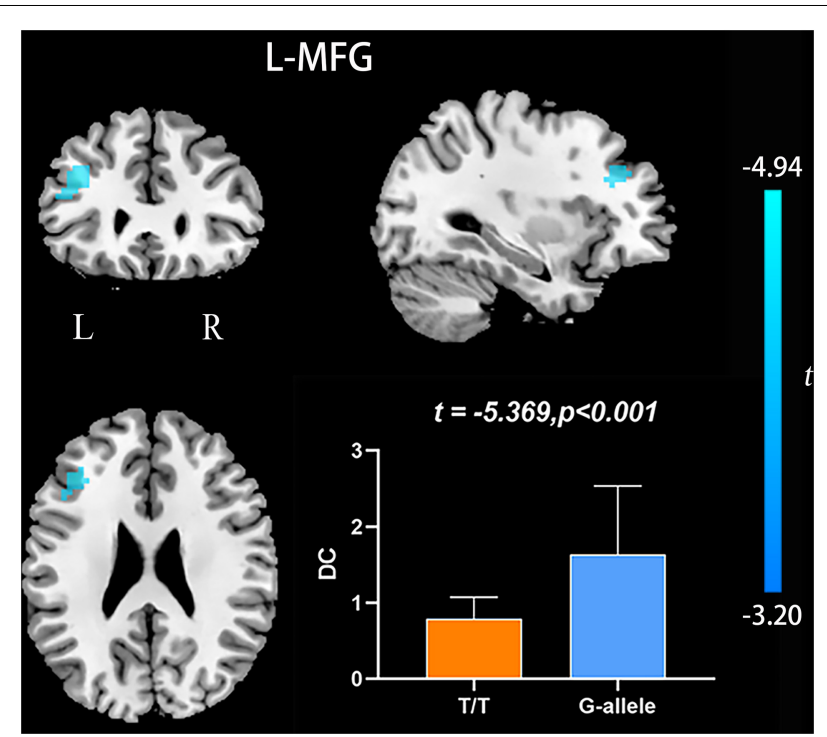

FIGURE 4 | DC differences between T/T carriers and G-allele carriers. Error bar represents the standard deviation. DC, degree centrality; L, left; R, right.

more promising metric for resting-state BOLD fMRI signal. Therefore, we believe that the results of ALFF and PerAF can complement each other to increase the credibility and persuasiveness of the results.
Regional homogeneity is a local index that reflects the local synchronization of the BOLD signal. The right cerebellar tonsil showed increased ReHo, while the right putamen showed decreased ReHo. The cerebellar tonsil is a part of the cerebellar vermis, which is crucial in regulating cognition and emotion (Stoodley and Schmahmann, 2009). Here, we found increased ReHo in the right cerebellum tonsil, which could be explained as a compensatory process. The compensatory hypothesis is considered to occur in the process of $\mathrm{AD}$ and in people at high risk of $\mathrm{AD}$ (Bookheimer et al., 2000). In addition, we found decreased ReHo in the right putamen in $\mathrm{T} / \mathrm{T}$ carriers relative to the $\mathrm{G}$ allele carriers. The putamen is a part of the striatum, projecting to the substantia nigra pars reticulata and internal pallidal segment. Cortico-basal ganglia-thalamocortical circuits are engaged in the control of movement, behavior, and cognition, as well as rewards and emotions (Obeso et al., 2014). This result may further confirm that $\mathrm{T} / \mathrm{T}$ allele may contribute to a variety of cognitive-behavioral declines.

Degree centrality is the embodiment of voxels' status and role in the whole brain network, and this measure represents the most representative and reliable indicator of local functional connections (Buckner et al., 2009). The left middle frontal gyrus showed decreased DC, which suggests a reduced significance of this region in the entire brain. Wen and colleagues found that the left middle frontal gyrus participated in word production and indicated that it might serve as a temporal perceptual information storage space (Wen et al., 2017). Andersson and colleagues argued that the left middle frontal gyrus is classified as the executive attention network. The left middle frontal gyrus'

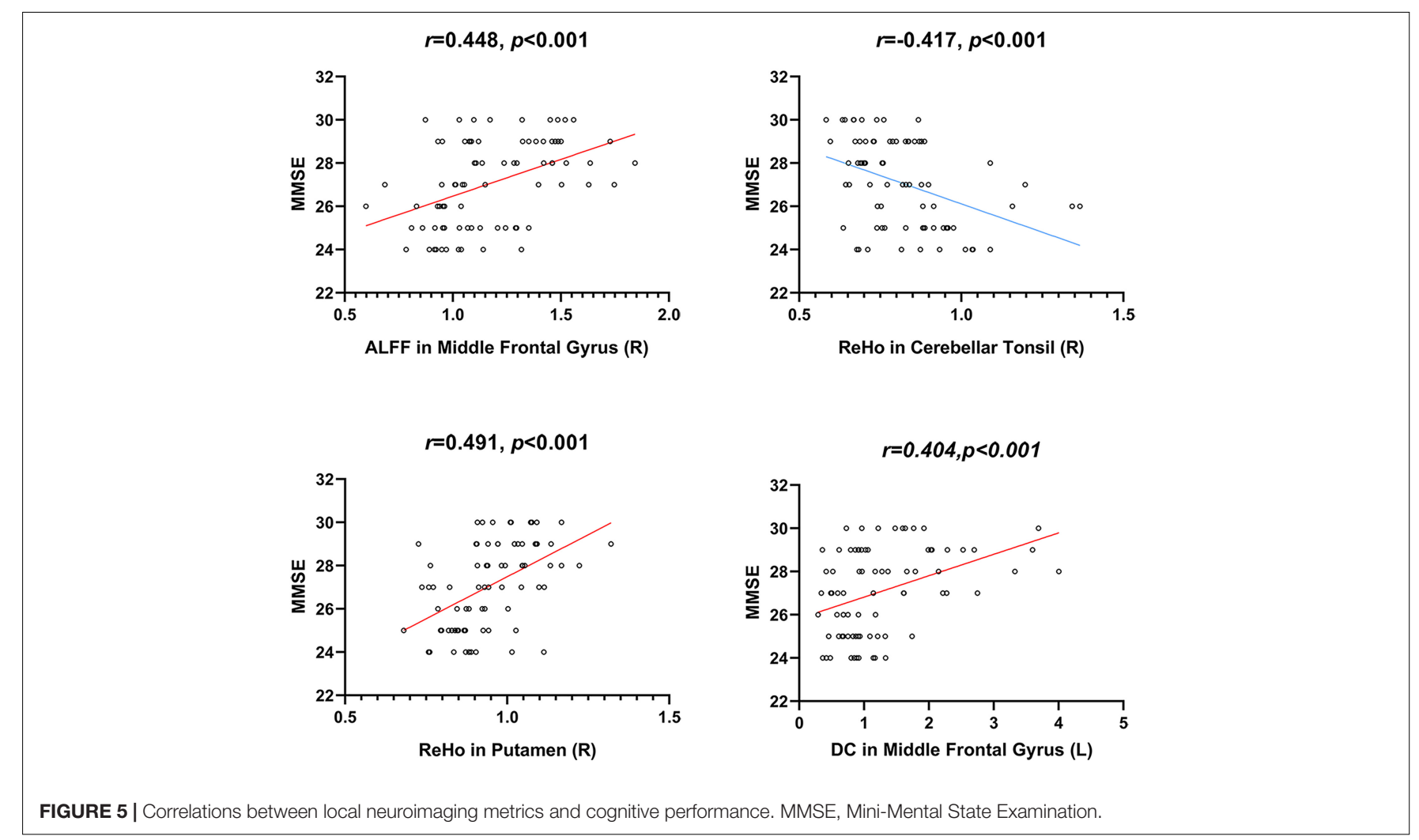


function is related to subtle attention disorders in the elderly (Andersson et al., 2009). Additionally, the middle frontal gyrus also engaged in an individual's literacy and numeracy (Koyama et al., 2017). Accordingly, we speculate that the reduced DC in the left middle frontal gyrus may indicate the decreased functional connectivity and may lead to attention, language, and cognition impairments in $\mathrm{T} / \mathrm{T}$ allele carriers.

These local indexes define the functional characteristics of the brain from distinct perspectives and show a step-bystep relationship. ALFF characterizes the spontaneous neural activity intensity of voxels, fALFF can get better default mode network patterns, PerAF has better test-retest reliability, both intra- and inter-scanners (Zhao et al., 2018), ReHo reveals the significance of voxels among nearest other voxels, and DC describes the importance of voxels in the entire brain. Applying these local metrics can identify regional abnormalities with greater sensitivity. For instance, An et al. (2013) found the group differences of ADHD patients and healthy controls using ReHo and ALFF; they found that group differences in ALFF and ReHo metrics were not the same. This finding intimate that these local metrics reveal changes in the local functions of the brain from different angles and complement each other.

Correlation analyses were conducted between neuro psychological scales and ROI-based mean imaging values. We found correlations between MMSE and imaging parameters in ALFF, ReHo, and DC. However, no significant correlation was found for other cognitive tests and other local metircs. MMSE is the most widely used simple cognitive function assessment scale in clinical practice, which has the advantages of simplicity, time-saving, and easy operation (Folstein et al., 1975). It is mainly used for the preliminary screening of various types of cognitive impairment and dementia. It can comprehensively, accurately, and quickly reflect the mental state and cognitive function of subjects. In this study, MMSE has a significant correlation with neuroimaging indicators, which proves that the neuropsychological scale and resting-state fMRI can simultaneously detect the cognitive impairment of subjects. Combining early cognitive tests and non-invasive resting-state functional magnetic resonance can provide early intervention and prevention for high-risk groups. The current study has several limitations. Firstly, the sample size is relatively modest. In the future, more subjects will be enrolled to increase statistical power. Additionally, no MRI data were collected during the follow-up period; thus, we cannot examine the T/T allele's ongoing effects on brain function in the elderly. Future longitudinal studies are needed to test whether the present method could be applied to supervise T/T allele carriers' brain alterations. Finally, due to the

\section{REFERENCES}

An, L., Cao, Q. J., Sui, M. Q., Sun, L., Zou, Q. H., Zang, Y. F., et al. (2013). Local synchronization and amplitude of the fluctuation of spontaneous brain activity in attention-deficit/hyperactivity disorder: a resting-state fMRI study. Neurosci. Bull. 29, 603-613. doi: 10.1007/s12264-013-1353-8

Andersson, M., Ystad, M., Lundervold, A., and Lundervold, A. J. (2009). Correlations between measures of executive attention and cortical thickness limitation of the scanning range, some subjects may not cover the whole brain. Among these subjects, the most inferior portion of the cerebellum is the common area that did not cover. We reported the result of ReHo in the cerebellum. Still, its position is not at the most inferior portion of the cerebellum, so it will not be affected by the scanning range.

\section{CONCLUSION}

In conclusion, this study demonstrated the effect of rs405509 on local brain function. T/T allele may serve as an independent risk factor that can influence brain function in different regions in non-demented aging. These preliminary findings reveal the significant role of $A P O E$ promoter polymorphism in the brain and provide novel insight into the risk of rs405509 polymorphism altering brain function and regulating $\mathrm{AD}$ development in nondemented aging.

\section{DATA AVAILABILITY STATEMENT}

The raw data supporting the conclusions of this article will be made available by the authors, without undue reservation.

\section{ETHICS STATEMENT}

The studies involving human participants were reviewed and approved by the Ethics Committee of The Affiliated Hospital of Qingdao University. The patients/participants provided their written informed consent to participate in this study.

\section{AUTHOR CONTRIBUTIONS}

JS, XL, and RZ designed the study. QW, XM, BH, and HG were responsible for performing the experiments and collecting the data. DW and HZ analyzed the cognitive and magnetic resonance data and wrote the manuscript. All authors contributed to the article and approved the submitted version.

\section{FUNDING}

This study was supported by a grant from the National Key R\&D Program of China (2018YFC1315200).

of left posterior middle frontal gyrus - a dichotic listening study. Behav. Brain Funct. BBF. 5:41. doi: 10.1186/1744-9081-5-41

Artiga, M. J., Bullido, M. J., Sastre, I., Recuero, M., Garcia, M. A., Aldudo, J., et al. (1998). Allelic polymorphisms in the transcriptional regulatory region of apolipoprotein E gene. FEBS Lett. 421, 105-108. doi: 10.1016/s0014-5793(97) 01543-3

Beyer, K., Lao, J., Gómez, M., Riutort, N., Latorre, P., Mate, J. L., et al. (2002). The Th1/E47cs-G apolipoprotein E (APOE) promoter allele is a risk factor 
for Alzheimer disease of very later onset. Neurosci. Lett. 326, 187-190. doi: 10.1016/s0304-3940(02)00355-5

Biswal, B., Yetkin, F. Z., Haughton, V. M., and Hyde, J. S. (1995). Functional connectivity in the motor cortex of resting human brain using echo-planar MRI. Magn. Reson. Med. 19, 537-541. doi: 10.1002/mrm.1910340409

Bookheimer, S. Y., Strojwas, M. H., Cohen, M. S., Saunders, A. M., Pericak-Vance, M. A., Mazziotta, J. C., et al. (2000). Patterns of brain activation in people at risk for Alzheimer's disease. N. Engl. J. Med. 343, 450-456. doi: 10.1056/ NEJM200008173430701

Buckner, R. L., Sepulcre, J., Talukdar, T., Krienen, F. M., Liu, H., Hedden, T., et al. (2009). Cortical hubs revealed by intrinsic functional connectivity: mapping, assessment of stability, and relation to Alzheimer's disease. J. Neurosci. 29, 1860-1873. doi: 10.1523/JNEUROSCI.5062-08.2009

Buckner, R. L., Snyder, A. Z., Sanders, A. L., Raichle, M. E., and Morris, J. C. (2000). Functional brain imaging of young, nondemented, and demented older adults. J. Cogn. Neurosci. 12(Suppl. 2), 24-34. doi: 10.1162/089892900564046

Chang, P., Li, X., Ma, C., Zhang, S., Liu, Z., Chen, K., et al. (2017). The effects of an APOE promoter polymorphism on human white matter connectivity during non-demented aging. J. Alzheimers Dis. JAD 55, 77-87. doi: 10.3233/ jad- 160447

Corbetta, M., Patel, G., and Shulman, G. L. (2008). The reorienting system of the human brain: from environment to theory of mind. Neuron 58, 306-324. doi: 10.1016/j.neuron.2008.04.017

Dai, Z., Yan, C., Li, K., Wang, Z., Wang, J., Cao, M., et al. (2015). Identifying and mapping connectivity patterns of brain network hubs in Alzheimer's disease. Cereb. Cortex (New York NY 1991) 25, 3723-3742. doi: 10.1093/cercor/bhu246

Farrer, L. A., Cupples, L. A., Haines, J. L., Hyman, B., Kukull, W. A., Mayeux, R., et al. (1997). Effects of age, sex, and ethnicity on the association between apolipoprotein E genotype and Alzheimer disease. A meta-analysis. APOE and Alzheimer Disease Meta Analysis Consortium. JAMA 278, 1349-1356. doi: 10.1001/jama.278.16.1349

Ferri, C. P., Prince, M., Brayne, C., Brodaty, H., Fratiglioni, L., Ganguli, M., et al (2005). Global prevalence of dementia: a Delphi consensus study. Lancet (Lond. Engl.) 366, 2112-2117. doi: 10.1016/S0140-6736(05)67889-0

Folstein, M. F., Folstein, S. E., and McHugh, P. R. (1975). "Mini-mental state". A practical method for grading the cognitive state of patients for the clinician. J. Psychiatr. Res. 12, 189-198. doi: 10.1016/0022-3956(75)90026-6

Fox, M. D., and Raichle, M. E. (2007). Spontaneous fluctuations in brain activity observed with functional magnetic resonance imaging. Nat. Rev. Neurosci. 8, 700-711. doi: 10.1038/nrn2201

Fox, M. D., Corbetta, M., Snyder, A. Z., Vincent, J. L., and Raichle, M. E. (2006). Spontaneous neuronal activity distinguishes human dorsal and ventral attention systems. Proc. Natl. Acad. Sci. U.S.A. 103, 10046-10051. doi: 10.1073/ pnas.0604187103

Friston, K. J., Williams, S., Howard, R., Frackowiak, R. S., and Turner, R. (1996). Movement-related effects in fMRI time-series. Magn. Reson. Med. 35, 346-355. doi: $10.1002 / \mathrm{mrm} .1910350312$

Gordon, N. G. (1972). The Trail Making Test in neuropsychological diagnosis. J. Clin. Psychol. 28, 167-169. doi: 10.1002/1097-4679(197204)28:2<167::aidjclp2270280212>3.0.c0;2-x

Heise, V., Filippini, N., Ebmeier, K. P., and Mackay, C. E. (2011). The APOE 44 allele modulates brain white matter integrity in healthy adults. Mol. Psychiatry 16, 908-916. doi: 10.1038/mp.2010.90

Hoptman, M. J., Zuo, X. N., Butler, P. D., Javitt, D. C., D’Angelo, D., Mauro, C. J., et al. (2010). Amplitude of low-frequency oscillations in schizophrenia: a resting state fMRI study. Schizophr. Res. 117, 13-20. doi: 10.1016/j.schres.2009.09.030

Ishiai, S., Sugishita, M., Ichikawa, T., Gono, S., and Watabiki, S. (1993). Clockdrawing test and unilateral spatial neglect. Neurology 43, 106-110. doi: 10.1212/ wnl.43.1_part_1.106

Jia, X. Z., Sun, J. W., Ji, G. J., Liao, W., Lv, Y. T., Wang, J., et al. (2020). Percent amplitude of fluctuation: a simple measure for resting-state fMRI signal at single voxel level. PLoS One 15:e0227021. doi: 10.1371/journal.pone.0227021

Knesevich, J. W., LaBarge, E., and Edwards, D. (1986). Predictive value of the Boston Naming Test in mild senile dementia of the Alzheimer type. Psychiatry Res. 19, 155-161. doi: 10.1016/0165-1781(86)90008-9

Koss, E., Ober, B. A., Delis, D. C., and Friedland, R. P. (1984). The Stroop colorword test: indicator of dementia severity. Int. J. Neurosci. 24, 53-61. doi: 10. 3109/00207458409079534
Koyama, M. S., O’Connor, D., Shehzad, Z., and Milham, M. P. (2017). Differential contributions of the middle frontal gyrus functional connectivity to literacy and numeracy. Sci. Rep. 7:17548. doi: 10.1038/s41598-017-17702-6

Küblböck, M., Woletz, M., Höflich, A., Sladky, R., Kranz, G. S., Hoffmann, A., et al. (2014). Stability of low-frequency fluctuation amplitudes in prolonged resting-state fMRI. NeuroImage 103, 249-257. doi: 10.1016/j.neuroimage.2014. 09.038

Lambert, J. C., Araria-Goumidi, L., Myllykangas, L., Ellis, C., Wang, J. C., Bullido, M. J., et al. (2002). Contribution of APOE promoter polymorphisms to Alzheimer's disease risk. Neurology 59, 59-66. doi: 10.1212/wnl.59.1.59

Lambert, J. C., Berr, C., Pasquier, F., Delacourte, A., Frigard, B., Cottel, D., et al. (1998a). Pronounced impact of Th1/E47cs mutation compared with -491 AT mutation on neural APOE gene expression and risk of developing Alzheimer's disease. Hum. Mol. Genet. 7, 1511-1516. doi: 10.1093/hmg/7.9.1511

Lambert, J. C., Brousseau, T., Defosse, V., Evans, A., Arveiler, D., Ruidavets, J. B., et al. (2000). Independent association of an APOE gene promoter polymorphism with increased risk of myocardial infarction and decreased APOE plasma concentrations-the ECTIM study. Hum. Mol. Genet. 9, 57-61. doi: $10.1093 / \mathrm{hmg} / 9.1 .57$

Lambert, J. C., Mann, D., Richard, F., Tian, J., Shi, J., Thaker, U., et al. (2005). Is there a relation between APOE expression and brain amyloid load in Alzheimer's disease? J. Neurol. Neurosurg. Psychiatry 76, 928-933. doi: 10.1136/ jnnp.2004.048983

Lambert, J. C., Pasquier, F., Cottel, D., Frigard, B., Amouyel, P., and ChartierHarlin, M. C. (1998b). A new polymorphism in the APOE promoter associated with risk of developing Alzheimer's disease. Hum. Mol. Genet. 7, 533-540. doi: $10.1093 / \mathrm{hmg} / 7.3 .533$

Lambert, J., Coyle, N., and Lendon, C. (2004). The allelic modulation of apolipoprotein $\mathrm{E}$ expression by oestrogen: potential relevance for Alzheimer's disease. J. Med. Genet. 41, 104-112. doi: 10.1136/jmg.2003.005033

Laws, S. M., Hone, E., Gandy, S., and Martins, R. N. (2003). Expanding the association between the APOE gene and the risk of Alzheimer's disease: possible roles for APOE promoter polymorphisms and alterations in APOE transcription. J. Neurochem. 84, 1215-1236. doi: 10.1046/j.1471-4159.2003. 01615.x

Liang, P., Liu, Y., Jia, X., Duan, Y., Yu, C., Qin, W., et al. (2011). Regional homogeneity changes in patients with neuromyelitis optica revealed by restingstate functional MRI. Clin. Neurophysiol. 122, 121-127. doi: 10.1016/j.clinph. 2010.05.026

Ma, C., Zhang, Y., Li, X., Chen, Y., Zhang, J., Liu, Z., et al. (2016a). The TT allele of rs405509 synergizes with APOE $\varepsilon 4$ in the impairment of cognition and its underlying default mode network in non-demented elderly. Curr. Alzheimer Res. 13, 708-717. doi: 10.2174/1567205013666160129100350

Ma, C., Zhang, Y., Li, X., Zhang, J., Chen, K., Liang, Y., et al. (2016b). Is there a significant interaction effect between apolipoprotein E rs405509 T/T and $\varepsilon 4$ genotypes on cognitive impairment and gray matter volume? Eur. J. Neurol. 23, 1415-1425. doi: 10.1111/ene.13052

Ma, X., Zheng, W., Li, C., Li, Z., Tang, J., Yuan, L., et al. (2019). Decreased regional homogeneity and increased functional connectivity of default network correlated with neurocognitive deficits in subjects with genetic high-risk for schizophrenia: a resting-state fMRI study. Psychiatry Res. 281:112603. doi: 10. 1016/j.psychres.2019.112603

Machulda, M. M., Jones, D. T., Vemuri, P., McDade, E., Avula, R., Przybelski, S., et al. (2011). Effect of APOE $\varepsilon 4$ status on intrinsic network connectivity in cognitively normal elderly subjects. Arch. Neurol. 68, 1131-1136. doi: 10.1001/ archneurol.2011.108

Maloney, B., Ge, Y. W., Petersen, R. C., Hardy, J., Rogers, J. T., Perez-Tur, J., et al. (2010). Functional characterization of three single-nucleotide polymorphisms present in the human APOE promoter sequence: differential effects in neuronal cells and on DNA-protein interactions. Am. J. Med. Genet. B Neuropsychiatr. Genet. 153B, 185-201. doi: 10.1002/ajmg.b.30973

Obeso, J. A., Rodriguez-Oroz, M. C., Stamelou, M., Bhatia, K. P., and Burn, D. J. (2014). The expanding universe of disorders of the basal ganglia. Lancet (Lond. Engl.) 384, 523-531. doi: 10.1016/S0140-6736(13)62418-6

Paakki, J. J., Rahko, J., Long, X., Moilanen, I., Tervonen, O., Nikkinen, J., et al. (2010). Alterations in regional homogeneity of resting-state brain activity in autism spectrum disorders. Brain Res. 1321, 169-179. doi: 10.1016/j.brainres. 2009.12.081 
Power, J. D., Barnes, K. A., Snyder, A. Z., Schlaggar, B. L., and Petersen, S. E. (2012). Spurious but systematic correlations in functional connectivity MRI networks arise from subject motion. NeuroImage 59, 2142-2154. doi: 10.1016/j. neuroimage.2011.10.018

Premi, E., Cauda, F., Gasparotti, R., Diano, M., Archetti, S., Padovani, A., et al. (2014). Multimodal FMRI resting-state functional connectivity in granulin mutations: the case of fronto-parietal dementia. PLoS One 9:e106500. doi: 10. 1371/journal.pone.0106500

Reiman, E. M., Chen, K., Alexander, G. E., Caselli, R. J., Bandy, D., Osborne, D., et al. (2004). Functional brain abnormalities in young adults at genetic risk for late-onset Alzheimer's dementia. Proc. Natl. Acad. Sci. U.S.A. 101, 284-289. doi: $10.1073 /$ pnas. 2635903100

Rosenberg, S. J., Ryan, J. J., and Prifitera, A. (1984). Rey auditory-verbal learning test performance of patients with and without memory impairment. J. Clin. Psychol. 40, 785-787. doi: 10.1002/1097-4679(198405)40:3<785::aidjclp2270400325>3.0.co;2-4

Sheridan, L. K., Fitzgerald, H. E., Adams, K. M., Nigg, J. T., Martel, M. M., Puttler, L. I., et al. (2006). Normative Symbol Digit Modalities Test performance in a community-based sample. Arch. Clin. Neuropsychol. 21, 23-28. doi: 10.1016/j. acn.2005.07.003

Shu, N., Li, X., Ma, C., Zhang, J., Chen, K., Liang, Y., et al. (2015). Effects of APOE promoter polymorphism on the topological organization of brain structural connectome in nondemented elderly. Hum. Brain Mapp. 36, 4847-4858. doi: 10.1002/hbm.22954

Stoodley, C. J., and Schmahmann, J. D. (2009). Functional topography in the human cerebellum: a meta-analysis of neuroimaging studies. NeuroImage 44, 489-501. doi: 10.1016/j.neuroimage.2008.08.039

van der Flier, W. M., Pijnenburg, Y. A., Fox, N. C., and Scheltens, P. (2011). Early-onset versus late-onset Alzheimer's disease: the case of the missing APOE varepsilon4 allele. Lancet Neurol. 10, 280-288. doi: 10.1016/S1474-4422(10) 70306-9

Wen, J., Yu, T., Liu, L., Hu, Z., Yan, J., Li, Y., et al. (2017). Evaluating the roles of left middle frontal gyrus in word production using electrocorticography. Neurocase 23, 263-269. doi: 10.1080/13554794.2017.1387275

West, R. (2000). In defense of the frontal lobe hypothesis of cognitive aging. J. Int. Neuropsychol. Soc. JINS 6, 727-729; discussion 30. doi: 10.1017/ s1355617700666109

Wu, T., Long, X., Zang, Y., Wang, L., Hallett, M., Li, K., et al. (2009). Regional homogeneity changes in patients with Parkinson's disease. Hum. Brain Mapp. 30, 1502-1510. doi: 10.1002/hbm.20622

Yan, C. G., Wang, X. D., Zuo, X. N., and Zang, Y. F. (2016). DPABI: data processing $\&$ analysis for (resting-state) brain imaging. Neuroinformatics 14, 339-351. doi: 10.1007/s12021-016-9299-4
Ye, S., Dunleavey, L., Bannister, W., Day, L. B., Tapper, W., Collins, A. R., et al. (2003). Independent effects of the $-219 \mathrm{G}>\mathrm{T}$ and epsilon 2/ epsilon 3/ epsilon 4 polymorphisms in the apolipoprotein $\mathrm{E}$ gene on coronary artery disease: the Southampton Atherosclerosis Study. Eur. J. Hum. Genet. 11, 437-443. doi: 10.1038/sj.ejhg.5200983

Zang, Y. F., He, Y., Zhu, C. Z., Cao, Q. J., Sui, M. Q., Liang, M., et al. (2007). Altered baseline brain activity in children with ADHD revealed by resting-state functional MRI. Brain Dev. 29, 83-91. doi: 10.1016/j.braindev.2006.07.002

Zang, Y., Jiang, T., Lu, Y., He, Y., and Tian, L. (2004). Regional homogeneity approach to fMRI data analysis. NeuroImage 22, 394-400. doi: 10.1016/j. neuroimage.2003.12.030

Zhang, Q., Wu, L., Du, C., Xu, K., Sun, J., Zhang, J., et al. (2020). Effects of an APOE promoter polymorphism on fronto-parietal functional connectivity during nondemented aging. Front. Aging Neurosci. 12:183. doi: 10.3389/fnagi. 2020.00183

Zhao, N., Yuan, L. X., Jia, X. Z., Zhou, X. F., Deng, X. P., He, H. J., et al. (2018). Intra- and inter-scanner reliability of voxel-wise whole-brain analytic metrics for resting state fMRI. Front. Neuroinform. 12:54. doi: 10.3389/fninf. 2018.00054

Zou, Q. H., Zhu, C. Z., Yang, Y., Zuo, X. N., Long, X. Y., Cao, Q. J., et al. (2008). An improved approach to detection of amplitude of low-frequency fluctuation (ALFF) for resting-state fMRI: fractional ALFF. J. Neurosci. Methods 172, 137-141. doi: 10.1016/j.jneumeth.2008.04.012

Zuo, X. N., Di Martino, A., Kelly, C., Shehzad, Z. E., Gee, D. G., Klein, D. F., et al. (2010). The oscillating brain: complex and reliable. NeuroImage 49, 1432-1445. doi: 10.1016/j.neuroimage.2009.09.037

Zuo, X. N., Ehmke, R., Mennes, M., Imperati, D., Castellanos, F. X., Sporns, O., et al. (2012). Network centrality in the human functional connectome. Cereb. Cortex (New York, NY 1991) 22, $1862-1875$.

Conflict of Interest: The authors declare that the research was conducted in the absence of any commercial or financial relationships that could be construed as a potential conflict of interest.

Copyright (c) 2021 Wu, Zhao, Gu, Han, Wang, Man, Zhao, Liu and Sun. This is an open-access article distributed under the terms of the Creative Commons Attribution License (CC BY). The use, distribution or reproduction in other forums is permitted, provided the original author(s) and the copyright owner(s) are credited and that the original publication in this journal is cited, in accordance with accepted academic practice. No use, distribution or reproduction is permitted which does not comply with these terms. 